\title{
Modeling the Orientation of AGN
}

\author{
Emily Down ${ }^{1}$ \\ ${ }^{1}$ Present address: Oxford Astrophysics, Keble Road, Oxford OX1 3RH, UK \\ ${ }^{2}$ NRC Herzberg Institute of Astrophysics, Victoria, Canada \\ Email: ejd@astro.ox.ac.uk
}

Keywords. galaxies: active, quasars: emission lines

The orientation of nineteen $0.8<z<2.3$ radio-loud quasars was measured using two independent methods. First, $\mathrm{H} \alpha$ was observed in the near IR using ISAAC at the VLT. The complex $\mathrm{H} \alpha$ emission lines were fitted with a range of models, some including emission from a flattened, extended accretion disk following Chen \& Halpern (1989). The models were compared using the Bayesian evidence, and the disk axis angles recovered. Second, models were fitted to the $\sim 10 \mathrm{MHz}$ to $20 \mathrm{GHz}$ radio spectral energy distributions (SEDs) to recover the jet angles, assuming that the emission is comprised of a broken power law arising from the radio lobes plus a Doppler-boosted core. We find the following:

1. From the $\mathrm{H} \alpha$ modeling, $95 \%$ of the sources were found to be compatible with the presence of a thin, circular, optically emitting accretion disk.

2. Kendall's $\tau$ coefficient shows a $1.8 \sigma$ correlation between the $178 \mathrm{MHz}$ radio luminosity of the quasars and the fitted disk angles, with a maximum fitted disk angle of $48^{\circ}$. This is consistent with predictions of the receding torus model in which more powerful radio sources have dusty tori with larger opening angles.

3. The disk and jet angles are correlated at $>3 \sigma$ significance by Kendall's $\tau$ (see Figure 1), so there is no evidence from this sample that the accretion disks are misaligned from the radio jets, which may occur in the wake of jet-triggering galaxy mergers.

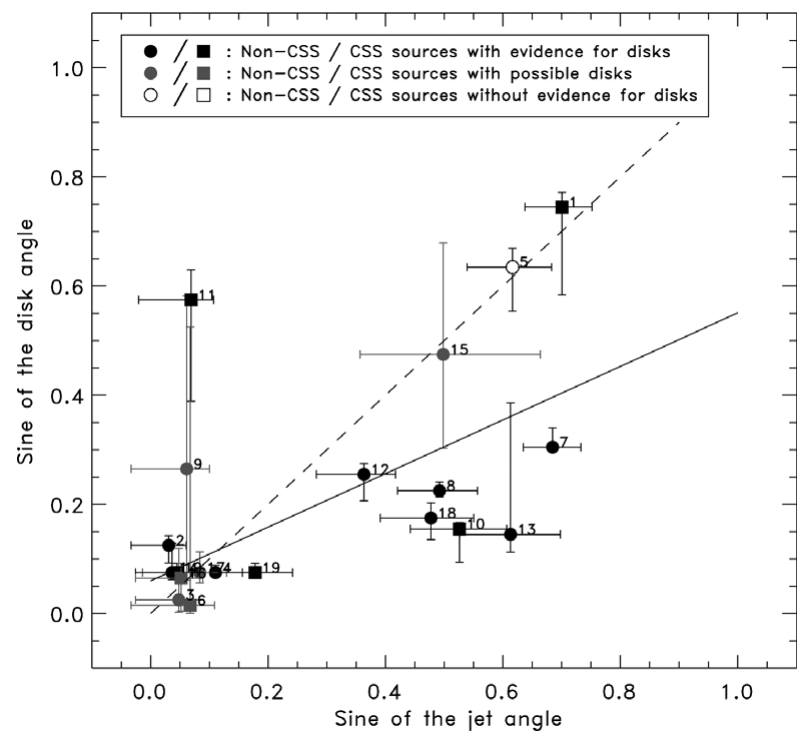

Figure 1. Disk angles from $\mathrm{H} \alpha$ emission plotted against jet angles fitted from radio SEDs.

\section{References}

Chen, K. \& Halpern, J. P. 1989, ApJ, 344, 115 